Research Paper:

\title{
Follow-up Comparison of the Effect of Eight-weeks Corrective Exercises With and Without Myofascial Release on Postural Kyphosis Deformity
}

\author{
Seyed Hossein Mousavi ${ }^{1}$ (D), Hooman Minoonejad²* ${ }^{*}$, Reza Rajabi ${ }^{3}$ (D) Foad Seidi $^{4}$ (D)
}

1. PhD. Student of Sports Injury and Corrective Exercises, Faculty of Physical Education and Sport Sciences, University of Tehran, Tehran, Iran.

2. Associate Professor, Department of Health and Sport Medicine, Faculty of Physical Education and Sport Sciences, University of Tehran, Tehran, Iran.

3. Professor, Department of Health and Sport Medicine, Faculty of Physical Education and Sport Sciences, University of Tehran, Tehran, Iran.

4. Associate Professor, Department of Health and Sport Medicine, Faculty of Physical Education and Sport Sciences, University Of Tehran, Tehran, Iran.

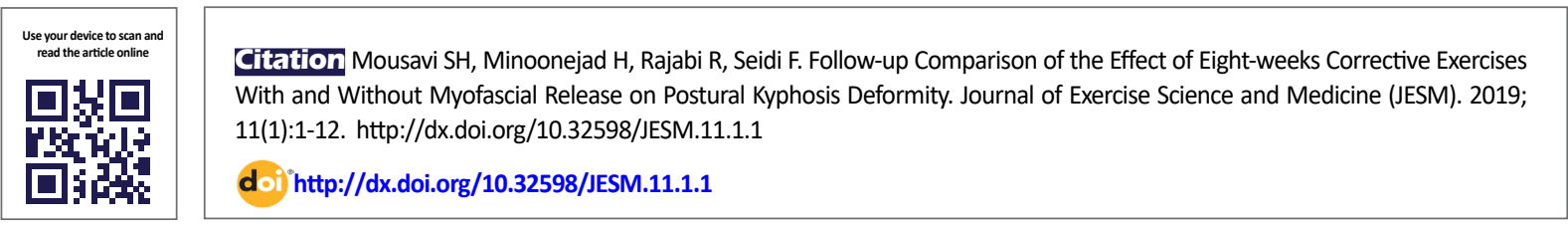

\section{(c) (i) (5)}

Article info:

Received: 26 Aug 2018

Accepted: 10 Nov 2018

Available Online: 01 Jan 2019

Keywords:

Myofascial release,

Corrective exercises, Postural hyperkyphosis

\section{A B S T RACT}

Introduction: The present research aimed to compare and follow-up the effect of 8-week corrective exercises with and without the myofascial release on functional kyphosis deformity in young adults.

Methods: It was a randomized controlled trial in the university laboratory. Forty-five male students suffering from abnormal hyperkyphosis deformity (kyphosis of more than $42^{\circ}$ ) were selected and randomly assigned to three groups: corrective exercises, combinational exercises (corrective and myofascial release), and control. The Mean $\pm S D$ age, height, and weight of the study sample were $22.12 \pm 2.88$ years, $175.44 \pm 6.80 \mathrm{~cm}$, and $71.21 \pm 7.31 \mathrm{~kg}$, respectively. The study groups did the exercises for 8 weeks, under the direct observation of the researchers. The kyphosis angle of the participants was measured using a flexible ruler, at the baseline, post-test, and follow-up (4 weeks after the end of the exercises) stages. The collected data were analyzed with repeated measures Analysis of Variance (ANOVA) and Analysis of Covariance (ANCOVA) tests.

Results: After 8 weeks of exercises, the degree of kyphosis in the post-test and follow-up stages was significantly lower than that in the pre-test, in both training groups. The results of the ANCOVA test demonstrated that the thoracic kyphosis degree in the combinational exercises group was significantly lower than that of the corrective exercises group $(P \leq 0.05)$, in the post-test and followup stages.

Conclusion: According to the results, the corrective exercises program in combination with the myofascial release is more effective than the corrective exercises alone, in correcting the thoracic kyphosis deformity.

\section{* Corresponding Author:}

Hooman Minoonejad, PhD.

Address: Department of Health and Sport Medicine, Faculty of Physical Education and Sport Sciences, University of Tehran, Tehran, Iran.

Tel: +98 (912) 3487425

E-mail:h.minoonejad@ut.ac.ir 


\section{Introduction}

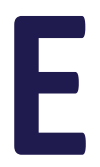

xcessive thoracic curvature or hyperkyphosis deformity is a prevalent postural abnormality and a major factor of upper quarter pathologies [1]. The prevalence of vertebral column anomalies is about $32 \%$ and $60 \%$ among the adults and the elderly people, respectively [2]. Rajabi et al. (2010) reported a higher rate of hyperkyphosis anomaly in men [3]. Other studies reported the prevalence rate of $15.3 \%$ in children aged 11 years [4], $38 \%$ in people aged $20-50$ years, and $35 \%$ in those aged 20-64 years for hyperkyphosis anomaly $[5,6]$.

The normal thoracic kyphosis angle of the Iranian population has been reported $42^{\circ}$ in people aged 18 to 30 years by Rajabi et al. (2008); angles higher than this are considered as hyperkyphosis [3]. Hyperkyphosis negatively affects the physical condition and the quality of physical activities [7, 8]. Individuals with hyperkyphosis walk slowly and have difficulty in climbing stairs. The risks of falling, fracture, and mortality are higher in these people, because of imbalance and the increased level of the general oscillation of their bodies $[7,9]$. This anomaly disrupts respiratory functions and daily activities, too [9]. Hyperkyphosis increases the biomechanical stresses applied to the vertebral column, the risk of osteoporosis in the vertebral column, and the compression fractures of the vertebrae $[10,11]$. Katzman et al. (2016) studied the relationship between hyperkyphosis and the prevalence of spine fracture. They reported that the prevalence of spine fracture increases by $22 \%$ for every $10^{\circ}$ increase in kyphosis [12].

Hyperkyphosis anomaly is treated and corrected through various methods, including manual therapy $[13,14]$, postural re-education $[15,16]$, using taping and orthoses [16], surgery, and therapeutic exercise [6, 17]. Therapeutic exercise is a common method. To manage kyphosis anomaly in patients, specialists often prescribe local therapeutic exercises, and recently, comprehensive and spine strengthening exercises $[12,18]$.

Sheikhhosseini et al. (2018) conducted a review study to examine the effectiveness of various corrective exercises for forward head posture anomaly (an uppercrossed syndrome anomaly). Results revealed desirable effectiveness of interventions for reducing the angle of forward head posture, but no specific finding has shown the effectiveness of these exercises for the related pains. The authors have recommended further studies to elucidate this matter [19].
According to the theoretical findings, there is a mutual relationship among postural anomalies in the upper quarter region. In other words, postural anomalies increase the incidence of kyphosis anomalies. This condition can cause changes along with the head and neck of people with hyperkyphosis [20]. According to Vladimir Genda, this anomaly is closely related to the other parts of the body in the form of a chain reaction and the separate local correction of each part cannot be scientifically justified [21]. Therefore, corrective exercises must be developed and implemented to manage the problems of patients with this anomaly. These exercises include strengthening the vertebral column, increasing the mobility of the vertebral column, and improving the alignment of the vertebral column, simultaneously.

Muscular tightness and the stiffness of connective tissue are common musculoskeletal problems that may emerge due to diseases, strokes, lack of physical activity, or scarring. The reduced flexibility of the soft tissue and motional restriction can alter movement patterns and cause musculoskeletal anomalies [22, 23]. Many methods used for maintaining the flexibility of muscles emphasize different stretching exercises [24]. Other techniques that are vastly employed for improving muscle flexibility are heat $[24,25]$, massaging [26], proprioceptive neuromuscular facilitation [27], muscle energy technique [28], and Myofascial Release (MFR) technique [29].

MFR technique is a manual technique used by the experts or the patient (self-myofascial release) to affect the fascia. Although it is not yet clear how these techniques work, their therapeutic effect has been clinically proven [30]. These techniques cause limited fascia stretching. Mild pressure for 90 to 120 seconds in the tissue increases flexibility in the limited tissue and the first release occurs afterward [31]. Tangible changes exist in the tissue structure and the end feel, that become palpable to the touch by the release of the superficial fascia. Next, the specialist applies more continuous and stable pressure without applying force downwards and towards the next resistive obstacle in the depth of the tissue, takes the tissue to a new range, and maintains it there; after some releases, the tissue becomes softer. The effects claimed for MFR techniques are similar to those reported for massing and soft tissue mobilization, including a change in blood circulation; the dilation of capillaries; and the stimulation of the nervous system, skin temperature, and metabolism. These changes increase the dilation and flexibility of the soft tissue, cause muscle relaxation, and reduce spasm and pain [30]. 
As noted before, a major complication of hyperkyphosis is stiffness or tightness created in the muscles of the segments in the vertebral region. If tightness and stiffness are resolved, the anomaly correction process will be facilitated and become more efficient. If this method is prescribed with an acceptable corrective protocol, it will have better results. The literature review showed that no study has examined the effectiveness of the simultaneous use of therapeutic exercises and MFR techniques for anomaly correction. Therefore, the present work aimed to examine the effectiveness of an 8-week protocol comprising corrective exorcises with and without MFR. This study aimed to answer this question: which method is more effective for correcting hyperkyphosis? Moreover, the persistence of the effects of these methods is examined and compared after four weeks of follow-up.

\section{Materials \& Methods}

This was a clinical trial examining the effect of corrective exercise protocol with and without MFR on hyperkyphosis in the experimental and control groups. The statistical population comprised non-athlete university male students with kyphosis postural anomaly $\left(\geq 42^{\circ}\right)$ aged 18 to 28 years in the Islamic Azad University of Zanjan, Iran. These students were identified by colleagues in physical education classes and introduced to the researchers. Then, based on the inclusion and exclusion criteria, the researchers selected 45 eligible participants and assigned them randomly to three groups: Corrective Exercises (CE), Corrective Exercises with MFR (CE+MFR), and control.

The inclusion criteria were as follows: The kyphosis postural anomaly of more than $42^{\circ}$ measured by a flexible ruler, the forward head of more than $46^{\circ}$, the forward shoulder of more than $52^{\circ}$ measured by imaging method, the age range of 18 to 28 years, and giving informed consent for voluntary participation. Also, the exclusion criteria were as follows: A history of fracture, surgery, or joint diseases in the vertebral column, shoulder girdle, or pelvis; the lack of lower cross syndrome; the lack of regular physical activity; joining a championship or sports teams; having special occupational conditions (which could exacerbate kyphosis and prevent postural correction at the workplace). The participants who were unwilling to continue the exercises, absent in two consecutive exercise sessions, absent in three sessions of the 8-week exercise program, and absent in measurement sessions were excluded from the study.
Initially, the participants were selected based on the inclusion and exclusion criteria. They signed the informed consent form and were randomly (by balloting) assigned to three groups (CE, CE+MFR, and control group). Then, they participated in the pre-test stage where the study variables (ie, kyphosis angle) were measured. This measurement was performed using a $50-\mathrm{cm}$ flexible ruler (IDIO). First, two spinous processes (T2, T12) were determined as the beginning and end of the thoracic kyphosis curve, then, the measurement was performed while the participants were in a standing position.

In the next step, standard demographic information (height, weight, and age) was measured using a measuring tape and digital scales and recorded in the special forms. The control group continued with their normal daily activity without special exercise, while the first experimental group received corrective exercises with MFR and the second experimental group received only corrective exercises for eight weeks (60-min sessions, 3 times per week) under the direct supervision of the researchers. The exercises were adapted from the comprehensive and supplementary exercises of Katzman (2016) [32].

The exercise program (Figure 1) included three main sections: vertebral column strengthening exercises, vertebral column motility enhancement exercises, and vertebral column alignment enhancement exercises. These exercises aimed to correct musculoskeletal anomalies related to hyperkyphosis and were developed in a multifaceted manner in three sections. The first section aimed to strengthen the stability and motility of the upper body, the lower trapezius, vertebral column extensor muscles, multifidus and transversus abdominis, the back and thigh extensors, the middle gluteal muscles for maintaining standing stability and to increase the motility of the vertebral column during rotations and extension of the vertebral column. Movements in this section included supine transversus abdominis on the roller (Figure 1 Exercise No. 1), quadruped arm and leg lift (Figure 1 Exercise No. 2), prone trunk lift to neutral (Figure 1 Exercise No. 3), side-lying thoracic rotation/extension (Figure 1 Exercise No. 4), and side-lying hip abduction/ external (lateral) rotation (Figure 1 Exercise No. 5).

Exercises in the second section aimed to increase the heart rate, core muscle temperature, the flexibility of the shoulder and rib cage, the stability of the torso, the motility of the shoulder and vertebral column, and also to strengthen the lower trapezius, serratus anterior, and scapula stability. Movements in this section included marching on the roller (Figure 1 exercise No. 6), unilateral overhead reaching on the roller (Figure 1 Exercise 


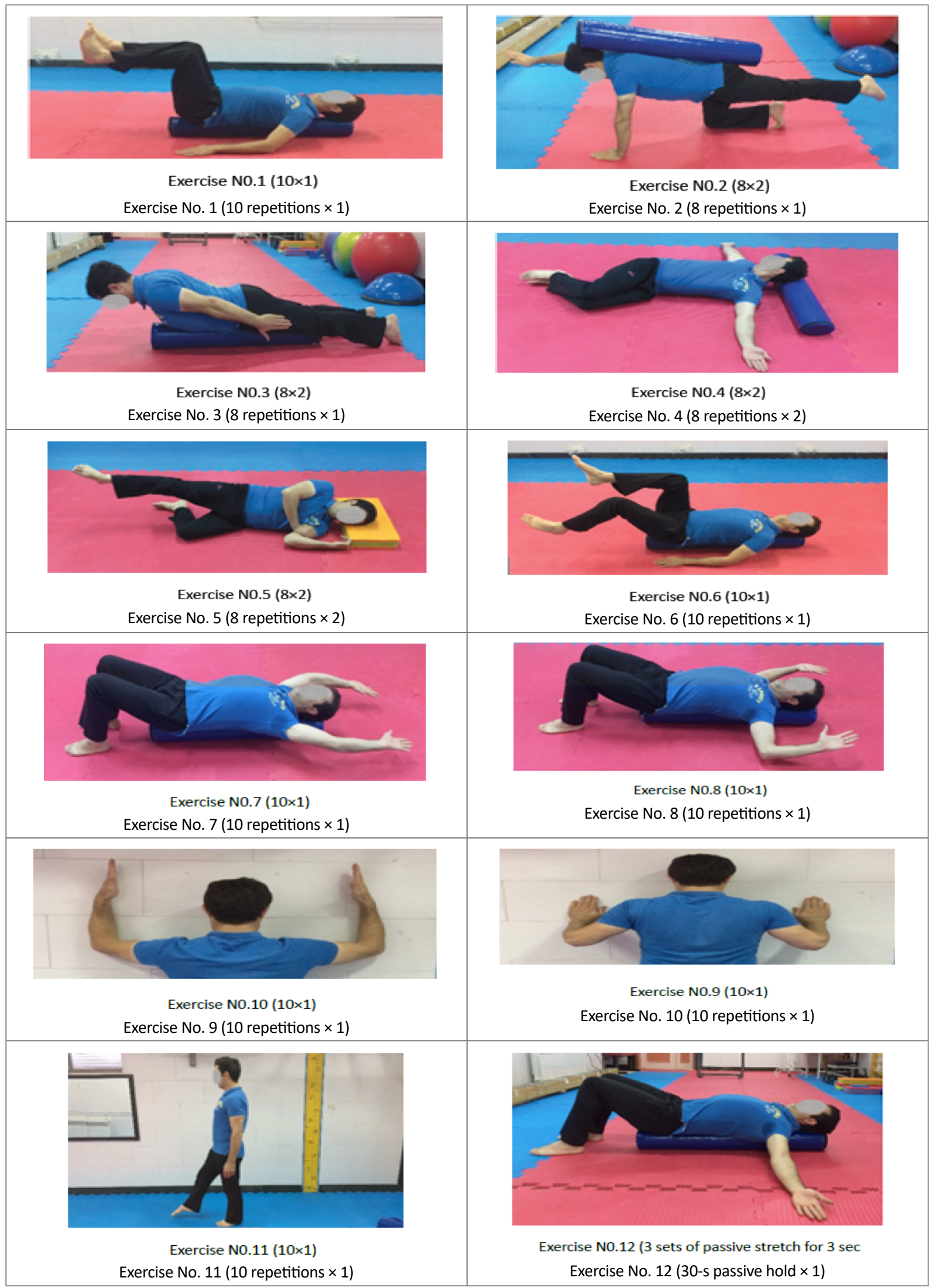




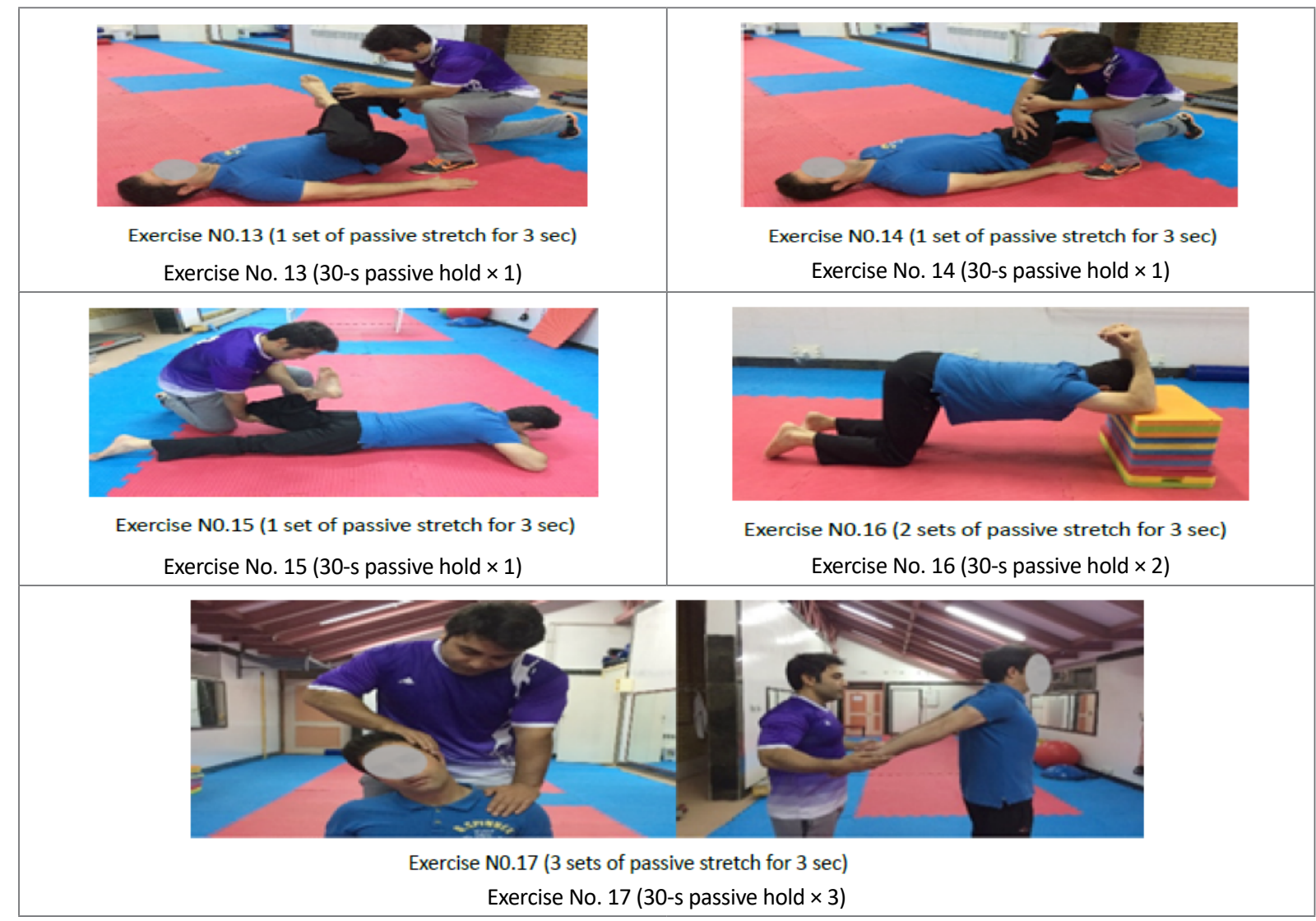

Figure 1. The corrective exercises program

Exercise Science and Medicine

No. 7), bilateral pull-down supine on the roller (Figure 1 Exercise No. 8), shoulder flexion or thoracic extension at the wall (Figure 1 Exercise No. 9), wall push-ups (Exercise No. 10), and single-leg stance (Figure 1 Exercise No. 11).

The third section included stretching the pectoralis major; increasing the expandability of the rib cage and anterior thoracic wall; increasing the length of posterior thigh muscles, gluteal, gastrocnemius, and soleus muscles; and lengthening iliacus quadriceps, the vertebral column, and the trapezius. The movements in this section included chest/spine stretching on the supine roller (Figure 1 Exercise No. 12), gluteal stretching (exercise No. 13), supine straight-leg raise (Figure 1 Exercise No. 14), prone hip/ quadriceps stretch (Figure 1 exercise No. 15), quadruped thoracic extension stretch (Figure 1 Exercise No. 16), and neck/chest stretch standing (Figure 1 Exercise No. 17).

The intensity of the exercises was increased during the study while emphasizing the good quality of movements. The participants maintained the intensity of exercises on the scores of 4 to 5 (70\%-80\%) based on the Burk's scale. They also received verbal and visual feedback that taught them to maintain and improve the neutral state of the vertebral column alignment during the exercise program. They were trained to keep their head aligned with the pelvis during the exercise programs and daily activities, so the head was remained aligned with the vertebral column in the neutral and stable positions while they were bending the knee or pelvis. In this study, the images of the ideal alignment of the vertebral column in the neutral state during functional activities (sitting, standing, moving from sitting to standing position, and lying down) were prepared and given to the participants.

In this study, the cross-hand release technique was used for MFR (Figure 2). Areas that underwent release included anterior thoracic (pectoral area) and back area, especially its upper part. To perform the releases, the researchers applied continuous mild pressure to the area; using the Duncan method, two hands were moved in opposite directions to the end of the available range (barrier) and were kept there until release. According to Duncan, the release of collagen bonds needs 90 to 120 seconds. Moreover, this technique was repeated twice in each region [31]. 


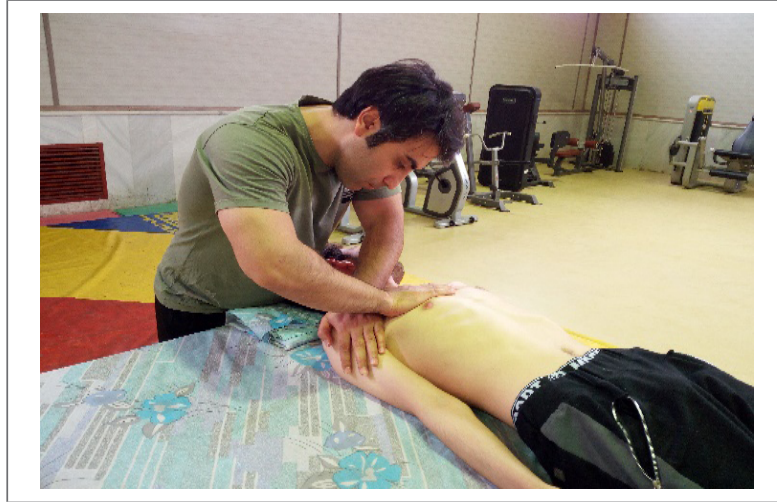

A. Cross-hand release technique of the anteriorupper chest

Figure 2. Cross-hand release technique

All the participants in the experimental and control groups were evaluated and their kyphosis angles were measured by a flexible ruler in the post-test stage (with the same procedure used in the pre-test). In each session, the corrective exercise group performed warmingup exercises while the hybrid group was receiving the MFR technique. After four weeks, the participants were again evaluated to measure the persistence of the effects of exercises on the follow-up.

The obtained data were analyzed using descriptive and inferential statistics in SPSS-20. After ensuring the normality of data distribution using the KolmogorovSmirnov test, the repeated measures Analysis of Variance (ANOVA) was used to specify within-group variations, and the Analysis of Covariance (ANCOVA) was employed to specify between-group variations. The test results were evaluated at the significance level of $95 \%(P<0.05)$.

\section{Results}

Table 1 presents the demographic information of the study sample. The results of the One-way ANOVA showed no significant difference among the three groups in terms of height, weight, and $\mathrm{BMI}(\mathrm{P}>0.05)$; thus, the study groups were homogeneous.

The repeated measures ANOVA was performed between the three groups to compare the thoracic kyphosis angle of different positions, in the pre-test, post-test, and follow-up stages (within-group variations). Table 2 reports the results; repeated measures ANOVA showed a significant difference in the within-groups variations of kyphosis angle in both experimental groups $(P<0.05)$.

Based on the significant results of ANOVA, the Bonferroni post-hoc test was used for the pairwise comparison

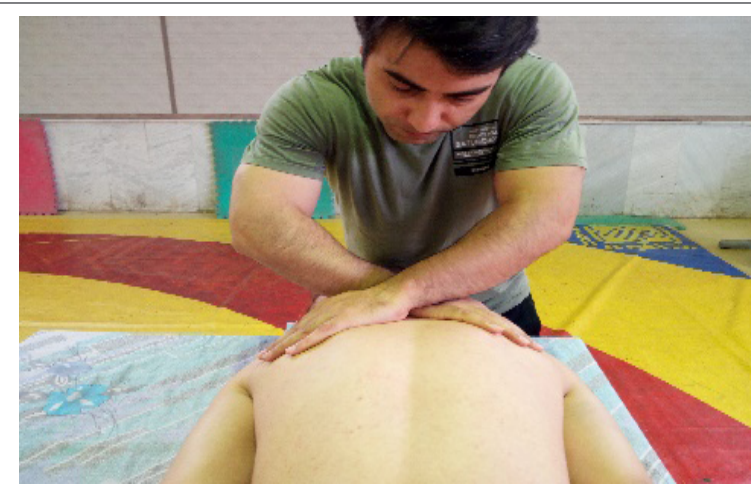

B. Cross-hand release technique of the upper back muscles

Exercise Science and Medicine

of variations in different experimental stages. Results indicated a significant difference in the kyphosis angle of the CE group (Mean difference=6.61, $P=0.001$ ) and the $\mathrm{CE} \pm \mathrm{MFR}$ group (Mean difference $=8.26, \mathrm{P}=0.001$ ). Also, the thoracic kyphosis was significantly reduced after 8 weeks. In the follow-up stage (four weeks after the exercise program), the level of thoracic kyphosis was significantly reduced in the CE group (Mean difference $=4.67$, $\mathrm{P}=0.001$ ) and the $\mathrm{CE} \pm \mathrm{MFR}$ group (Mean difference $=6.52$, $\mathrm{P}=0.001$ ) compared with the pre-test stage.

The ANCOVA was used to compare the effects of corrective exercises and corrective exercises with MFR on the thoracic kyphosis angle, in the post-test and followup. The pre-test was regarded as the covariate in these tests. Results showed a significant difference between the three groups, both in the post-test stage $(P=0.001$, $F=263.13$, partial $\eta^{2}=0.93$ ) and in the follow-up stage $\left(P=0.001, F=68.84\right.$, partial $\left.\eta^{2}=0.78\right)$.

Considering the significance of repeated measures ANCOVA, the Bonferroni post-hoc test was used for the pairwise comparison of variations (Table 3). Results showed that the thoracic kyphosis angle of the CE \pm MFR group was significantly less than that of the CE and control groups, in the post-test stage $(\mathrm{P}<0.05)$. Moreover, the level of kyphosis in the CE group was significantly less than that of the control group $(P<0.05)$.

Bonferroni test results showed that the thoracic kyphosis angle of the CE \pm MFR group was significantly less than that of the CE and control groups, in the follow-up stage $(P<0.05)$. Moreover, the level of kyphosis in the CE group was significantly less than that of the control group $(P<0.05)$. 
Table 1. Demographic information of study sample $(n=42)$

\begin{tabular}{|c|c|c|c|c|}
\hline Variable & Group & Mean $\pm S D$ & $\mathrm{~F}^{*}$ & $\mathrm{P} * *$ \\
\hline & CE & $22.38 \pm 3.04$ & & \\
\hline \multirow[t]{3}{*}{ Age (y) } & $C E \pm M F R$ & $22.07 \pm 2.89$ & 0.08 & 0.91 \\
\hline & Control & $21.93 \pm 2.71$ & & \\
\hline & $\mathrm{CE}$ & $175.69 \pm 7.85$ & & \\
\hline \multirow[t]{3}{*}{ Height (cm) } & $C E \pm M F R$ & $175.64 \pm 6.86$ & 0.04 & 0.95 \\
\hline & Control & $175 \pm 5.7$ & & \\
\hline & CE & $70.92 \pm 0.52$ & & \\
\hline \multirow[t]{3}{*}{ Weight (kg) } & $C E \pm M F R$ & $70 \pm 5.76$ & 0.51 & 0.60 \\
\hline & Control & $72.73 \pm 7.66$ & & \\
\hline & $\mathrm{CE}$ & $23.04 \pm 2.99$ & & \\
\hline \multirow[t]{2}{*}{ BMI $\left(\mathrm{kg} / \mathrm{m}^{2}\right)$} & $C E \pm M F R$ & $22.78 \pm 2.66$ & 0.50 & 0.60 \\
\hline & CE & $23.87 \pm 3.36$ & & \\
\hline
\end{tabular}

CE: Corrective Exercises; MFR: Myofascial Release; * One-way ANOVA; ** P $\leq 0.05$

Exercise Science and Medicine

Table 2. Results of repeated measured ANOVA for the variations of kyphosis angle between experimental stages in the study groups

\begin{tabular}{|c|c|c|c|c|c|}
\hline Group & Stage & Mean \pm SD & $\mathbf{F}$ & $\mathbf{P}$ & $\eta^{2 *}$ \\
\hline \multirow[t]{3}{*}{ CE } & Pre-test & $47.73 \pm 2.97$ & 96.67 & $0.001 * *$ & 0.89 \\
\hline & Post-test & $41.11 \pm 1.77$ & & & \\
\hline & Follow-up & $43.04 \pm 1.99$ & & & \\
\hline \multirow[t]{3}{*}{ CE $\pm M F R$} & Pre-test & $48.68 \pm 2.59$ & 208.33 & $0.001^{* *}$ & 0.94 \\
\hline & Post-test & $40.42 \pm 1.59$ & & & \\
\hline & Follow-up & $42.16 \pm 2.28$ & & & \\
\hline \multirow[t]{3}{*}{ Control } & Pre-test & $47.97 \pm 2.99$ & 0.161 & 0.852 & 0.01 \\
\hline & Post-test & $48.09 \pm 2.71$ & & & \\
\hline & Follow-up & $47.98 \pm 2.59$ & & & \\
\hline
\end{tabular}

* Partial eta squared shows the effect size (the percent of post-test variance explained by the independent variable); **P $\leq 0.05$

The Standard Error of Measurement (SEM) was calculated to compute the reliability index of the kyphosis angle. The SEM is an index for the precision of measurement, the stability, and the reliability of the test. It shows the distribution of the repeated measurements of one person with the same device around the actual score [33]. To this end, the kyphosis angle of 15 people was measured three times with a 3-day interval; the SEM calculation represented the measurement precision of $0.6^{\circ}$.

\section{Discussion}

The present randomized controlled trial was conducted in 45 young men: 15 in the control group, 15 in the hybrid exercise group (corrective exercises \pm MFR), and
15 in the corrective exercise group (1-hour sessions, 3 times per week, for 8 weeks, under the direct supervision of the researchers). A significant difference, in terms of reduced kyphosis angle, was observed among the hybrid exercise and the corrective exercise groups, compared with the control group. Results showed that the kyphosis angle in the post-test was reduced by an average of $8^{\circ}$ and $6^{\circ}$ in the hybrid exercise and the corrective exercise groups, respectively, compared with the pre-test stage $(P=0.001)$. Moreover, the results of ANCOVA indicated that the hybrid program had a more significant effect than the corrective exercise program. The effect size of the hybrid and the corrective exercise programs was 0.94 and 0.89 , receptively; these are considered large effect sizes, based on Cohen's d [34]. Furthermore, according to the SEM of $0.6^{\circ}$, the measure- 
Table 3. Bonferroni test results for the effects of exercises on kyphosis angle between the study groups

\begin{tabular}{|c|c|c|c|c|}
\hline Stages & Group (I) & Group (J) & Average Differences (I-J) & P* \\
\hline \multirow{4}{*}{ Post-test } & \multirow{2}{*}{$\mathrm{CE} \pm \mathrm{MFR}$} & $\mathrm{CE}$ & -1.31 & 0.006 \\
\hline & & Control & -8.14 & 0.001 \\
\hline & \multirow{2}{*}{ CE } & Control & -6.83 & 0.001 \\
\hline & & $\mathrm{CE} \pm \mathrm{MFR}$ & 1.31 & 0.006 \\
\hline \multirow{4}{*}{ Follow-up } & \multirow{2}{*}{$\mathrm{CE} \pm \mathrm{MFR}$} & $\mathrm{CE}$ & -1.49 & 0.044 \\
\hline & & Control & -6.27 & 0.001 \\
\hline & \multirow{2}{*}{ CE } & Control & -4.77 & 0.001 \\
\hline & & $C E \pm M F R$ & 1.49 & 0.044 \\
\hline
\end{tabular}

ment index has high precision in this study. The mean kyphosis angle of the control group did not change significantly in the post-test stage $(\mathrm{P}=0.852)$. Compared with the post-test, the level of kyphosis angle did not significantly change in the follow-up (four weeks after the exercises); it shows the persistence effects of both exercise protocols.

Fascia is a specialized connective tissue covering the entire body. Histologically, it is formed of cells and extracellular matrix (consisting of collagen, elastin, and reticulin). Fascia demonstrates the characteristics of viscoelastic materials in response to force. Its visco property leads to permanent deformity, and its elastic property returns it to its resting length over time. When stress is applied to the fragile collagen cross-links, its length changes permanently, compared with the resting length. The MFR techniques are applied in the form of continuous and usually mild pressure (for 90-120 s), leading to the stretching of the elastic components of the elastic collagen matrix and continues until the release is stopped. During the release, the matrix material flows, causing the middle viscous material to turn from the cell state into a gel state [30]. It seems that the MFR technique used in this study (ie, cross-hand technique) breaks the cross-sectional connections (plastic deformity) and stimulates the matrix material (affecting elastic properties), thereby, enhancing the flexibility of the fascia tissue [30].

Increased muscular flexibility caused by MFR can also be explained neurobiologically. Ruffini ending is one of the neurons in the fascia and specifically responds to touching forces and lateral stretches. It seems that the Ruffini stimulations reduce the activity of the sympathetic nervous system. This finding is in line with most of the clinical findings that suggest techniques that slowly affect deep tissues and can apply relaxing effects both locally and whole body [35]. Researchers have also shown that the free ends of interneurons (types 3 and 4 that comprise $80 \%$ of sensory neurons) serve as mechanoreceptors and react to mechanical forces or pressure in the fascia [36]. Gelhourne (1967) showed that deep manual pressure, especially if exerted slowly and continuously on the fascia, stimulates intermediate and Ruffini mechanoreceptors, bodily fluids, and the local metabolism of the tissue, and also causes muscle and mental relaxation [37].

The stimulation of mechanoreceptors during the MFR technique changes the proprioception pulses that enter the central nervous system. These changes alter the motor units of the tissues. Moreover, the intense stimulation of intermediate receptors can directly affect the autonomic nervous system, thus, it leads to the exit of plasma from the veins, alters the viscosity of the extracellular matrix, and turns the matrix material from the cell state into the gel state [35]. These beneficial effects of MFR strengthen the back extensor, deep muscles, and the central area of the body. These changes lead to a significant improvement that was observed in the hybrid exercise program.

The implementation of a strong exercise protocol of the vertebral column plus stretching exercises can reduce the angle of kyphosis, in hyperkyphosis [12]. The muscles of the torso contribute to the control of movements and stability of the vertebral column. Deep muscles and the central area of the body play a vital role in the stability and control of inter-vertebrate movements; they stabilize the vertebral column even in dynamic states. In the exercise protocol of this study, it was attempted to strengthen deep and central muscles as well as the back extensor muscles. The other objectives of our exercise protocol were the stretching of pectoral muscles, and increasing the expandability of the rib cage, 
anterior thoracic wall, and the mobility of the vertebral column. In this exercise program, we attempted to perform body mass exercises with the closed motion chain on the foam roller [32]. According to previous studies, a series of changes occurs in skeletal muscles after performing the resistance exercises. These changes increase the total contractile protein, especially in myosin cords, the amount and strength of connective tissue cords, the density of capillaries, the number of cords, and the longitudinal division of muscle cords, which consequently increase muscle endurance. Also, the stretching exercises coordinate agonist and antagonist muscles [38]. Therefore, the exercises performed in this study elongated thoracic anterior muscles and strengthened back and central muscles, thereby, reduced the anomalies.

In this study, further effectiveness was observed for reducing the kyphosis angle, after the combination of corrective exercises with MFR. This correction was $8^{\circ}$, and demonstrates the further effectiveness of hybrid exercises, compared with corrective exercises and the previous studies with other techniques. Comprehensive strength and stretching exercises in combination with MFR released the myofascial tissue and coordinated agonist and antagonist muscle groups, thus, significantly decreased the kyphosis angle, compared with corrective exercises alone. Moreover, postural exercises were taught through verbal and visual feedback methods to the experimental groups, and they used these strategies during the course and daily activities. They were instructed to keep the neutral alignment of the vertebral column in all conditions and improve this state during the exercises. They were also trained to keep their head aligned with the pelvis during exercise programs and daily activities, so the head was remained aligned with the vertebral column in the neutral and stable positions while they were bending the knee or pelvis [32].

Katzman et al. (2017) used a similar protocol for women and men with 60 years of age and the thoracic kyphosis of more than $40^{\circ}$ [39]. After six months of exercise, the results of measurement with kyphometer showed that thoracic kyphosis was significantly reduced $\left(3.8^{\circ}\right)$; this result is consistent with the present findings. In their study, the effect of age on the degree of thoracic kyphosis can be considered as the main reason for the small reduction of kyphosis angle. Moreover, some participants had idiopathic bone kyphosis that could have affected their results. Seydi et al. (2014) examined the effect of comprehensive and local corrective exercises on kyphosis. They concluded that compared with local exercises, comprehensive exercises led to the further reduction of thoracic kyphosis [20]. Czaprowsky et al. (2014) aimed to perform the active correction of the kyphosis state by training and promoting the participants: "not to slouch." They observed a significant improvement in the kyphosis and lordosis of 249 individuals aged 10-14 years; this improvement was only performed by training how to actively keep the back straight while sitting and standing [40]. In a review study, Bensal et al. (2014) reported that studies on the effects of exercise intervention on kyphosis typically have low quality or small sample sizes, and most of them reported moderate effectiveness for improving the kyphosis angle [41].

Based on the significant reduction of kyphosis angle in both experimental groups, compared with the control group, and its more significant reduction in the hybrid exercise group than in the corrective exercise group, it can be concluded that the corrective exercises protocol in combination with MFR had desirable effectiveness for decreasing the back kyphosis angle. Various studies have emphasized the importance and applicability of MFR techniques. However, this was the first study that used this technique in combination with corrective exercises on hyperkyphosis and reported its high effectiveness. Therefore, we recommend using MFR, as an important and effective factor, in combination with corrective exercises to resolve thoracic kyphosis anomalies. On the other hand, the exercises had high persistence, because the effectiveness of the interventions persisted on for a 4-week follow-up, in both groups.

In this study, the researchers tried to correct anomalies in young people after reviewing all the relevant studies in Iran and other countries, considering the review studies of the effects of exercise interventions on kyphosis anomalies (which mostly recommended the simultaneous use of strengthening and stretching exercises in combination with postural exercises), and by combining these exercises with MFR as a recommended effective method. The exercises performed in this study were similar to the comprehensive and supplementary exercises of Katzman and were based on a set of comprehensive corrective exercises developed in previous studies for hyperkyphosis correcting (Katzman, 2017). While implementing the MFR, the comprehensive protocol of strength exercises, and simultaneous stretching exercises, for the vertebral column, the researchers also used postural exercises to enhance the effectiveness of exercises for the hybrid exercise group. Thus, the hybrid exercises had a lasting effect and significantly reduce kyphosis, compared with the corrective exercises. 


\section{Conclusion}

According to the results of the present study, the proposed corrective exercise protocol in combination with the MFR reduces thoracic hyperkyphosis, and its effect persists on the follow-up. Thus, researchers and specialists are recommended to use MFR, as a novel and effective method, in combination with corrective exercises to correct anomalies.

\section{Ethical Considerations}

\section{Compliance with ethical guidelines}

This study has been approved by the National Ethics Committee of Biomedical Research (Code: IR.UT.SPORT. REC.1397.004).

\section{Funding}

This research did not receive any grant from funding agencies in the public, commercial, or non-profit sectors.

\section{Authors' contributions}

All authors were equally contributed in preparing this article.

\section{Conflict of interest}

The authors declared no conflict of interest.

\section{Reference}

[1] Hertling D, Kessler RM. Management of common musculoskeletal disorders: Physical therapy principles and methods. Philadelphia: Lippincott Williams \& Wilkins; 2006.

[2] Liu S, Diebo BG, Henry JK, Smith JS, Richard H, Matthew E Cunningham et al. The benefit of nonoperative treatment for adult spinal deformity: identifying predictors for reaching a minimal clinically important difference. The Spine Journal. 2016; 16(2):210-8. [DOI:10.1016/j.spinee.2015.10.043] [PMID]

[3] Rajabi R, Latifi S. Soft curvature of the spinal cord (kyphosis) and lumbar (lordosis) of Iranian men and women. Journal Research in Sport Sciences. 2010; 28(18):13-30.

[4] Hazebroek-Kampschreur A, Hofman A, van Dijk AP, Van Linge B. Prevalence of trunk abnormalities in eleven-year-old schoolchildren in Rotterdam, The Netherlands. Journal of Pediatric Orthopaedics. 1992; 12(4):480-4. [DOI:10.1097/01241398-199207000-00012] [PMID]

[5] Griegel-Morris P, Larson K, Mueller-Klaus K, Oatis CA. Incidence of common postural abnormalities in the cervical, shoulder, and thoracic regions and their association with pain in two age groups of healthy subjects. Physical Therapy. 1992; 72(6):425-31. [DOI:10.1093/ptj/72.6.425] [PMID]

[6] Carter N, Khan K, Petit M, et al. Results of a 10 week community based strength and balance training programme to reduce fall risk factors: A randomised controlled trial in 65-75 year old women with osteoporosis. British Journal of Sports Medicine. 2001; 35(5):348-51. [DOI:10.1136/bjsm.35.5.348] [PMID] [PMCID]

[7] Kado DM, Huang M-H, Barrett-Connor E, Greendale GA. Hyperkyphotic posture and poor physical functional ability in older communitydwelling men and women: The Rancho Bernardo study. The Journals of Gerontology Series A: Biological Sciences and Medical Sciences. 2005; 60(5):633-7. [DOI:10.1093/gerona/60.5.633] [PMID] [PMCID]

[8] Kado DM, Huang MH, Karlamangla AS, Barrett-Connor E, Greendale GA. Hyperkyphotic posture predicts mortality in older communitydwelling men and women: A prospective study. Journal of the American Geriatrics Society. 2004; 52(10):1662-7. [DOI:10.1111/j.15325415.2004.52458.x] [PMID]

[9] Katzman W, Cawthon P, Hicks GE, Vittinghoff E, Shepherd J, Cauley $\mathrm{JA}$, et al. Association of spinal muscle composition and prevalence of hyperkyphosis in healthy community-dwelling older men and women Journals of Gerontology Series A: Biomedical Sciences and Medical Sciences. 2011; 67(2):191-5. [DOI:10.1093/gerona/glr160] [PMID] [PMCID]

[10] Hongo M, Miyakoshi N, Shimada Y, Sinaki M. Association of spinal curve deformity and back extensor strength in elderly women with osteoporosis in Japan and the United States. Osteoporosis International. 2012; 23(3):1029-34. [DOI:10.1007/s00198-011-1624-z] [PMID]

[11] Kado DM, Huang MH, Karlamangla AS, Cawthon P, Katzman W, Hillier TA, et al. Factors associated with kyphosis progression in older women: 15 years' experience in the study of osteoporotic fractures. Journal of Bone and Mineral Research. 2013; 28(1):179-87. [DOI:10.1002/ jbmr.1728] [PMID] [PMCID]

[12] Katzman WB, Vittinghoff E, Kado DM, Schafer AL, Wong SS, Gladin A, et al. Study of Hyperkyphosis, Exercise and Function (SHEAF) protocol of a randomized controlled trial of multimodal spine-strengthening exercise in older adults with hyperkyphosis. Physical therapy. 2016 96(3):371-81. [DOI:10.2522/ptj.20150171] [PMID] [PMCID]

[13] Twomey LT. A rationale for the treatment of back pain and joint pain by manual therapy. Physical Therapy. 1992; 72(12):885-92. [DOI:10.1093/ptj/72.12.885] [PMID]

[14] Edmondston S, Singer K. Thoracic spine: Anatomical and biomechanical considerations for manual therapy. Manual Therapy. 1997; 2(3):132-43. [DOI:10.1054/math.1997.0293] [PMID]

[15] Birbaumer N, Flor H, Cevey B, Dworkin B, Miller NE. Behavioral treatment of scoliosis and kyphosis. Journal of Psychosomatic Research. 1994; 38(6):623-8. [DOI:10.1016/0022-3999(94)90060-4]

[16] Bennell K, Khan K, McKay H. The role of physiotherapy in the prevention and treatment of osteoporosis. Manual Therapy. 2000; 5(4):198213. [DOI:10.1054/math.2000.0369] [PMID]

[17] Sinaki M. Critical appraisal of physical rehabilitation measures after osteoporotic vertebral fracture. Osteoporosis International. 2006; 17(11):1702. [DOI:10.1007/s00198-006-0139-5]

[18] Seidi F, Rajabi R, Alizadeh MH, Daneshmandi H. [The effect of a 10week selected corrective exercise program on postural thoracic kyphosis deformity (Persian)]. Journal of Sport Medicine. 2013; 5(1):5-22 [DOI:10.22059/JSMED.2013.32159] 
[19] Sheikhhoseini R, Shahrbanian S, Sayyadi P, O’Sullivan K. Effectiveness of therapeutic exercise on forward head posture: A systematic review and meta-analysis. Journal of Manipulative and Physiological Therapeutics. 2018; 41(6):530-9. [DOI:10.1016/j.jmpt.2018.02.002] [PMID]

[20] Seidi F, Rajabi R, Ebrahimi E, Alizadeh MH, Minoonejad H. The efficiency of corrective exercise interventions on thoracic hyperkyphosis angle. Journal of Back and Musculoskeletal Rehabilitation. 2014; 27(1):7-16. [DOI:10.3233/BMR-130411] [PMID]

[21] Page P, Frank C, Lardner R. Assessment and treatment of muscle imbalance: The Janda approach. $1^{\text {st }}$ edition. Illinois: Human Kinetics, Inc.; 2010.

[22] Prentice WE, Kaminski TW. Rehabilitation techniques for sports medicine and athletic training. New York: McGraw-Hill; 2004.

[23] Reed BV, Ashikaga T, Fleming BC, Zimny NJ. Effects of ultrasound and stretch on knee ligament extensibility. Journal of Orthopaedic \& Sports Physical Therapy. 2000; 30(6):341-7. [DOI:10.2519/ jospt.2000.30.6.341] [PMID]

[24] Knight CA, Rutledge CR, Cox ME, Acosta M, Hall SJ. Effect of superficial heat, deep heat, and active exercise warm-up on the extensibility of the plantar flexors. Physical Therapy. 2001; 81(6):1206-14. [DOI:10.1093/ptj/81.6.1206] [PMID]

[25] Funk D, Swank AM, Adams KJ, Treolo D. Efficacy of moist heat pack application over static stretching on hamstring flexibility. The Journal of Strength \& Conditioning Research. 2001; 15(1):123-6. [DOI:10.1519/1533-4287(2001)0152.0.CO;2]

[26] Barlow A, Clarke R, Johnson N, Seabourne B, Thomas D, Gal J. Effect of massage of the hamstring muscle group on performance of the sit and reach test. British journal of sports medicine. 2004; 38(3):349-51. [DOI:10.1136/bjsm.2002.003673] [PMID] [PMCID]

[27] Funk DC, Swank AM, Mikla BM, Fagan TA, Farr BK. Impact of prior exercise on hamstring flexibility: A comparison of proprioceptive neuromuscular facilitation and static stretching. The Journal of Strength \& Conditioning Research. 2003; 17(3):489-92. [DOI:10.1519/1533-4287(2003)0172.0.CO;2]

[28] Spernoga SG, Uhl TL, Arnold BL, Gansneder BM. Duration of maintained hamstring flexibility after a one-time, modified hold-relax stretching protocol. Journal of Athletic Training. 2001; 36(1):44.

[29] Kain J, Martorello L, Swanson E, Sego S. Comparison of an Indirect Tri-planar Myofascial Release (MFR) technique and a hot pack for increasing range of motion. Journal of Bodywork and Movement Therapies. 2011; 15(1):63-7. [DOI:10.1016/j.jbmt.2009.12.002] [PMID]

[30] Ezadkhah S, Naseri N, Maroufi N, Kachili Y, Shabdin H. [Comparison of myofascial release and muscle energy technique effects on hamstring muscle flexibility (Persian)]. Journal of Modern Rehabilitation. 2016; 9(5):131-9.

[31] Duncan R. Myofascial release. Illinois: Human Kinetics, Inc.; 2014

[32] Katzman W, Vittinghoff E, Kado D, Lane N, Ensrud K, Shipp K. Thoracic kyphosis and rate of incident vertebral fractures: The fracture intervention trial. Osteoporosis International. 2016; 27(3):899-903. [DOI:10.1007/s00198-015-3478-2] [PMID] [PMCID]

[33] Hakimipour M, Rajabi R, Minoonejad H, Soltani A. [Reliability of foot photo box in measuring selected footprint indices (Persian)]. Quarterly Journal of Applied Life Sciences in Sport. 2016; 4(8):122-114.

[34] Cohen J. Statistical power analysis for the behavioral sciences. $2^{\text {n }} d$ edition. NJ, Erlbaum: Hillsdale; 1988.
[35] Schleip R. Fascial plasticity-a new neurobiological explanation: Part 1. Journal of Bodywork and Movement Therapies. 2003 7(1):11-9. [DOI:10.1016/S1360-8592(02)00067-0]

[36] Mitchell JH, Schmidt RF. Cardiovascular reflex control by afferent fibers from skeletal muscle receptors. Handbook of Physiology. The Cardiovascular System Peripheral Circulation and Organ Blood Flow. 1983; 3(pt 2):623-58.

[37] Gellhorn E. Principles of autonomic-somatic integrations. University of Minnesota Press: Minneapolis; 1967.

[38] Jang H J, Kim M J, Kim SY. Effect of thorax correction exercises on flexed posture and chest function in older women with agerelated hyperkyphosis. Journal of Physical Therapy Science. 2015; 27(4):1161-4. [DOI:10.1589/jpts.27.1161] [PMID] [PMCID]

[39] Katzman WB, Vittinghoff E, Lin F, Schafer A, Long RK, Wong S, et al. Targeted spine strengthening exercise and posture training program to reduce hyperkyphosis in older adults: results from the Study of Hyperkyphosis, Exercise, and Function (SHEAF) randomized controlled trial. Osteoporosis International. 2017; 28(10):2831-41. [DOI:10.1007/s00198-017-4109-x] [PMID] [PMCID]

[40] Czaprowski D, Pawłowska P, Stoliński $Ł$, Kotwicki T. Active selfcorrection of back posture in children instructed with 'straighten your back' command. Manual Therapy. 2014; 19(5):392-8. [DOI:10.1016/j.math.2013.10.005] [PMID]

[41] Bansal S, Katzman WB, Giangregorio LM. Exercise for improving age-related hyperkyphotic posture: A systematic review. Archives of Physical Medicine and Rehabilitation. 2014; 95(1):129-40. [DOI:10.1016/j.apmr.2013.06.022] [PMID] [PMCID] 
This Page Intentionally Left Blank 